\title{
Blocking Behaviors of Crosstalk-Free Optical Banyan Networks on Vertical Stacking
}

\author{
Xiaohong Jiang, Member, IEEE, Hong Shen, Md. Mamun-ur-Rashid Khandker, Student Member, IEEE, and \\ Susumu Horiguchi, Senior Member, IEEE
}

\begin{abstract}
Banyan networks are attractive for constructing directional coupler (DC)-based optical switching networks for their small depth and self-routing capability. Crosstalk between optical signals passing through the same DC is an intrinsic drawback in DC-based optical networks. Vertical stacking of multiple copies of an optical banyan network is a novel scheme for building nonblcoking (crosstalk-free) optical switching networks. The resulting network, namely vertically stacked optical banyan (VSOB) network, preserves all the properties of the banyan network, but increases the hardware cost significantly. Though much work has been done for determining the minimum number of stacked copies (planes) required for a nonblocking VSOB network, little is known on analyzing the blocking probabilities of VSOB networks that do not meet the nonblcoking condition (i.e., with fewer stacked copies than required by the nonblocking condition). In this paper, we analyze the blocking probabilities of VSOB networks and develop their upper and lower bounds with respect to the number of planes in the networks. These bounds depict accurately the overall blocking behaviors of VSOB networks and agree with the conditions of strictly nonblocking and rearrangeably nonblocking VSOB networks respectively. Extensive simulation on a network simulator with both random routing and packing strategy has shown that the blocking probabilities of both strategies fall nicely within our bounds, and the blocking probability of packing strategy actually matches the lower bound. The proposed bounds are significant because they reveal the inherent relationships between blocking probability and network hardware cost in terms of the number of planes, and provide network developers a quantitative guidance to trade blocking probability for hardware cost. In particular, our bounds provide network designers an effective tool to estimate the minimum and maximum blocking probabilities of VSOB networks in which different routing strategies may be applied. An interesting conclusion drawn from our work that has practical applications is that the hardware cost of a VSOB network can be reduced dramatically if a predictable and almost negligible nonzero blocking probability is allowed.
\end{abstract}

Index Terms-Banyan networks, blocking probability, multistage interconnection networks (MINs), switching networks, vertical stacking.

\section{INTRODUCTION}

$\mathbf{I}$ T IS expected that users of telecommunication services such as Internet, Web browsing, and tele-education will increase dramatically. This has greatly increased the demand for high bandwidth and high capacity communication systems. Optical

Manuscript received September 19, 2001; revised June 18, 2002; approved by IEEE/ACM TRANSACTIONS ON NETWORKING Editor N. McKeown. This work was supported in part by Grant-In-Aid Scientific Research, Ministry of Education and Science, Japan.

The authors are with the Graduate School of Information Science, Japan Advanced Institute of Science and Technology (JAIST), Tatsunokuchi, Ishikawa 923-1292, Japan (e-mail: jiang @ jaist.ac.jp; shen@ jaist.ac.jp; hori@ jaist.ac.jp). Digital Object Identifier 10.1109/TNET.2003.820425

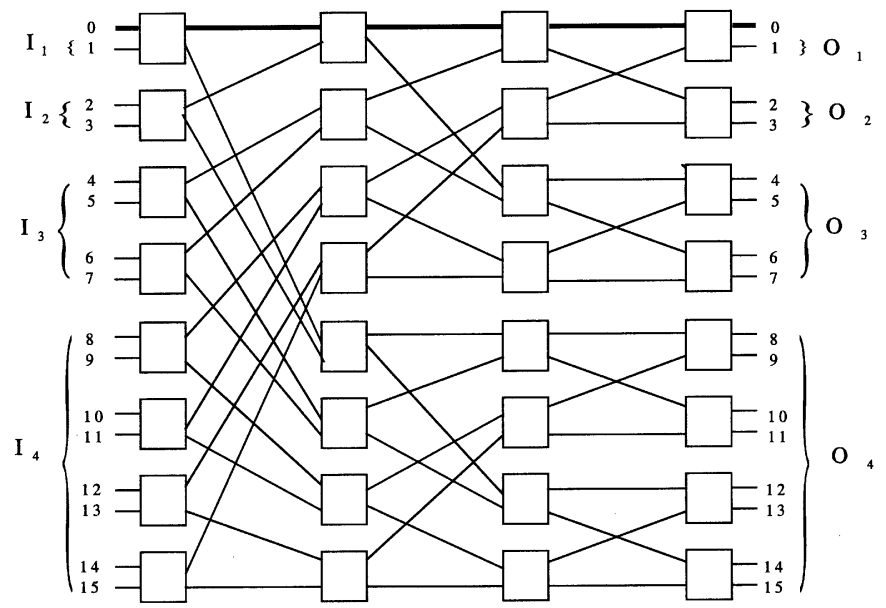

Fig. 1. A $16 \times 16$ banyan network (even number of stages).

networks are considered as a promising candidate to meet this demand. Optical switching network is an essential part in an optical network, which has the capability of switching huge data at an ultra-high speed. The $2 \times 2$ switching element (SE) in optical switching networks is usually a directional coupler (DC) that is created by manufacturing two waveguides close to each other [6]. The cross (bar) state of a DC can be implemented by applying a suitable voltage (no voltage) to it. Crosstalk in DC is a major shortcoming in DC-based optical networks, which occurs between two signals carried by the two waveguides of a DC [3], [6]. When two optical signals pass through a DC, a portion of optical power in one waveguide will be coupled into the other unintended waveguide and this undesirable coupling is called first-order crosstalk, which will then propagate stage by stage, introducing high order crosstalks at a reduced magnitude. Due to the stringent bit-error-rate (BER) requirement of optical transmission facilities, elimination of crosstalk has become an important issue for making optical networks work properly. By ensuring that only one signal passes through a DC at a time, the first-order crosstalk can be eliminated. This provides a costeffective solution to the crosstalk problem.

A banyan network [5] and its topological equivalence (e.g., baseline, omega) [8], [12] are a class of important switching networks and they are generally referred to as banyan networks [10]. A typical $N \times N$ banyan network consists of $\log _{2} N$ stages, each containing $N / 22 \times 2$ switches, and the link connections between adjacent stages are implemented by recursively applying the butterfly interconnection pattern, as shown in Fig. 1.

A banyan network has a simple switch setting ability (selfrouting) and also a small number of SEs along a path between 


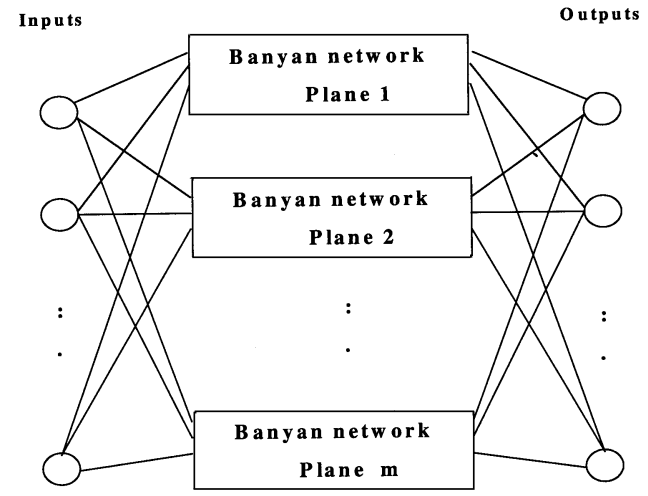

Fig. 2. Creating nonblocking (crosstalk-free) network based on the vertical stacking scheme.

an input-output pair. These characteristics have made banyan networks attractive for constructing DC-based optical switching networks because loss and attenuation of an optical signal are proportional to the number of couplers that the optical signal passes through. In this paper, we will focus on the optical banyan networks that are free of first-order crosstalk in SEs (we refer to this as crosstalk-free hereafter). It is the crosstalk-free constraint that makes the analysis of optical banyan networks different from that for electronic ones. In banyan networks, there is a unique path between an input-output pair and hence when two connections intend to use the same link, one of them will be blocked. This is called link-blocking. There is, however, another type of blocking in optical banyan networks. If adding the connection causes some paths including the new one to violate the crosstalk-free constraint, the connection cannot be added even if the path is available. We refer to this second type of blocking as crosstalk-blocking. Since the crosstalk-free constraint requires that only one signal is allowed to passes through a SE at a time, thus it has a larger contribution to the overall blocking probability than that of link-blocking.

Vertical stacking of multiple copies of a optical banyan network is a novel scheme for constructing nonblocking (crosstalk-free) optical switching networks with neither increasing the number of stages nor sacrificing the self-routing property of the banyan network [9]. Fig. 2 illustrates the vertical stacking scheme. We use VSOB to denote vertically stacked optical banyan networks and $\operatorname{VSOB}(N, m)$ to denote an $N \times N$ VSOB network that has $m$ stacked copies (planes) of an $N \times N$ banyan network. Numerous results are available for VSOB networks, such as [7], [11], [13], [14], and their main focus has been on determining the minimum number of planes required for nonblocking VSOB networks. These results indicate that the vertical stacking scheme, although is attractive, requires a prohibitively high hardware cost for building a nonblocking VSOB network.

Analysis of blocking probability of a network that does not meet the hardware requirement for nonblocking is an effective approach to studying network performance. Two well-known probabilistic models for multistage interconnection networks (MINs) were developed by Lee [15] and Jacobaeus [16], respectively, for analysis of the blocking behavior of the Clos network [17]. Based on the treatments similar to those established by Lee and Jacobaeus, a lot of work has been done on performance analysis of banyan networks [8], [12], [18]-[20]. However, most existing probabilistic results are for electronic networks only. That is, they address only link-blocking, not crosstalk-blocking. To our knowledge, no analytical result is available for estimating the blocking probabilities of optical switching networks built on vertical stacking of banyan network.

In this paper, we provide comprehensive analysis of the blocking probabilities in VSOB networks whose hardware costs (number of stacked planes) do not meet the nonblocking condition. The main contributions of our work are the following.

- We extend the available probabilistic methods for analysis of electronic networks where only link-blocking is concerned to analyze the blocking probability of a VSOB network where the crosstalk-blocking is the main contribution to blocking probability.

- We establish the upper and lower bounds on blocking probability of a VSOB network. These bounds reveal the inherent relationships between the blocking probability and the network hardware cost in terms of the number of vertically stacked planes. They are consistent with the conditions of strictly nonblocking and rearrangeably nonblocking VSOB networks, respectively, so they can nicely describe the overall blocking behaviors of VSOB networks.

- We develop a network simulator to verify our bounds and perform extensive simulation on it. It is shown that the proposed bounds are correct and are consistent with simulation results. The results from further simulation with both random routing and packing strategy show that the blocking probability of packing strategy matches with the proposed lower bound and blocking probability of random routing is nicely bounded by the lower and upper bounds.

The results of our work have a number of important applications: Our upper and lower bounds can guide network designers to determine the effects of reduction in the number of planes on the overall blocking behaviors in VSOB networks and to find desirable tradeoffs between hardware cost and blocking probability according to needs. In particular, the lower bound can guide the network designers to estimate the minimum blocking probability of a VSOB network and also the blocking probability in a network when packing strategy is applied. On the other hand, the upper bound can guide the network designers to estimate the maximum blocking probability of a VSOB network in which different routing strategies may be applied. Our work draws an interesting conclusion that we can reduce the hardware cost of a VSOB network dramatically by allowing a predictable and almost negligible nonzero blocking probability. Because this blocking probability is negligibly small in many cases, the significant cost-savings in network hardware becomes very appealing for practical designs and applications of VSOB networks.

The rest of the paper is organized as follows. Section II briefly describes the deterministic condition for strictly nonblocking VSOB networks. The derivation of the upper and lower bounds are presented in Sections III and IV, respectively. Section $\mathrm{V}$ compares the simulation results and theoretical findings. Section VI summarizes the results of the paper. 
Before we proceed to the Section II, we need to give some definitions and assumptions that will facilitate our discussions. In this paper, we consider the banyan networks that support one-to-one communication. We define the blocking probability to be the probability that a feasible connection request is blocked, where a feasible connection request is a connection request between an idle input port and an idle output port of the network.

Following the same assumption as in [15], [16], [20], [21] on probabilistic analysis of MINs, we neglect the correlation among signals arriving at input (output) ports and consider that the statuses (busy or idle) of individual input (output) ports in the network are independent. This assumption is justified by the fact that the correlation among signals at inputs (outputs), though exists for fixed communication patterns, becomes negligible for arbitrary communication patterns in large-size networks, which is the trend of future optical switching networks that can switch huge data at high speeds.

\section{Deterministic CONDITION FOR STRICTLY NONBLOCKING}

In this section, we briefly describe the deterministic condition for the strictly nonblocking VSOB network that is obtained based on worst-case analysis. We also evaluate the probability that the worst-case scenario occurs to motivate the work of this paper.

Due to their topological symmetry, all paths in a banyan network have the same property in terms of blocking. To study the blocking probability, we can arbitrarily select an input and an output in the network and set up a connection between them. Through out this paper, we will select the path between the first input and the first output and try to set up a connection between them. We call the path between this input-output pair the tagged path. All the SEs on the tagged path are called tagged SEs. The stages of SEs are numbered from left (stage 1) to right (stage $\log N^{1}$ ). For a tagged path, an input intersecting set $I_{i}=\left\{2^{i-1}, 2^{i-1}+1, \ldots, 2^{i}-1\right\}$ at stage $i$ is defined as the set of all inputs that intersect a tagged SE at stage $i$. Likewise, an output intersecting set $O_{i}=\left\{2^{i-1}, 2^{i-1}+1, \ldots, 2^{i}-1\right\}$ associated with stage $i$ is the set of all outputs that intersect a tagged SE at stage $\log N-i+1,1 \leq i \leq \log N$.

Ideally, we are interested in an optical network that is nonblocking and crosstalk-free. This can be achieved at the cost of extra hardware. For a VSOB network, the following theorem gives the deterministic condition for strictly nonblocking [13].

Theorem 1: $\operatorname{VSOB}(N, m)$ is strictly nonblocking if the following condition is true:

$$
m \geq \begin{cases}2 \sqrt{N}-1, & \text { if } \log N \text { is even } \\ \frac{3}{2} \sqrt{2 N}-1, & \text { if } \log N \text { is odd }\end{cases}
$$

The above result was obtained based on worst-case analysis. That is, to find the maximum possible number of connections that will conflict the tagged path and let each of these connections block a distinct plane. From Theorem 1, it is clear that the hardware cost for a strictly nonblocking VSOB network is high.

It is interesting to find out the probability that the worst-case scenario could occur. Let the probability that an input (output) port is busy be $r$ and denote by $P_{\text {worst }}$ the probability that the

${ }^{1}$ In this paper log means logarithm to the base 2 . worst-case scenario occurs. $P_{\text {worst }}$ is then given in the following lemma under the assumption that statuses of individual input (output) ports are independent.

Lemma 1: In an $N \times N$ optical banyan network, we have

$$
\begin{aligned}
P_{\text {worst }}= & \left(\prod_{i=1}^{(1 / 2) \log N}\left(r \times \frac{\frac{N}{2^{i-1}}-\sqrt{N}}{N-1}\right)^{2^{i-1}}\right)^{2}, \\
\text { if } \log N \text { is even; } & \left(\prod_{i=1}^{(1 / 2)(\log N-1)}\left(r \times \frac{\frac{N}{2^{i-1}}-\sqrt{2 N}}{N-1}\right)^{2^{i-1}}\right)^{2} \\
& \cdot\left(r \times \frac{\sqrt{\frac{N}{2}}}{N-1}\right)^{\sqrt{N / 2}}, \text { if } \log N \text { is odd. }
\end{aligned}
$$

Proof: For the case when $\log N$ is even (Fig. 1), we further define input set $I_{\text {even }}$ and output set $O_{\text {even }}$ as

$$
I_{\text {even }}=\bigcup_{i=1}^{((1 / 2)) \log N} I_{i}, \quad O_{\text {even }}=\bigcup_{i=1}^{((1 / 2)) \log N} O_{i} .
$$

Under the constraint of crosstalk-free, the worst-case scenario of conflicts on the tagged path is when all inputs in set $I_{i}$ are destined for the outputs in set $O_{(\log N-i+1)}$ and all outputs in set $O_{i}$ are originated from the inputs in set $I_{(\log N-i+1)}$ for $1 \leq i \leq(1 / 2) \log N$. Thus, the maximum number of conflicts with the tagged path is determined by both the connections from set $I_{\text {even }}$ and the connections destined for set $O_{\text {even }}$. There are $2^{i-1}$ connections from $I_{i}$, each of which can reach any of the $N-1$ outputs. In order to block the target path, a connection from $I_{i}$ must be terminated at an output in $\bigcup_{j=i}^{\log N} O_{\log N-j+1}$. In the worst-case where the connections between $I_{\text {even }}$ and $O_{\text {even }}$ are excluded to maximize the number of conflicts with the tagged path, the connection from $I_{i}$ will not go to $O_{\text {even }}=\bigcup_{j=1}^{(1 / 2)) \log N} O_{j} \subset \bigcup_{j=i}^{\log N} O_{\log N-j+1}$ for $1 \leq$ $i \leq(1 / 2) \log N$. Since $\left|\bigcup_{j=i}^{\log N} O_{\log N-j+1}\right|=N / 2^{i-1}-1$, $\left|O_{\text {even }}\right|=\sqrt{N}-1$, and the probability that an input (output) link is busy is $r$. The probability that a connection from $I_{i}$ blocks the tagged path but not destined for $O_{\text {even }}$ is thus

$$
\beta_{i}=r \times \frac{\frac{N}{2^{i-1}}-\sqrt{N}}{N-1}, \quad 1 \leq i \leq \frac{1}{2} \log N .
$$

So, by (4) and $\left|I_{i}\right|=\left|O_{i}\right|=2^{i-1}$, the probability that all connections coming from $I_{\text {even }}$ and going to $O_{\text {even }}$ simultaneously share no paths and block the tagged path is given by (2).

The proof for the case when $\log N$ is odd is the same as for the case when $\log N$ is even, after taking out all connections passing through the middle stage on the tagged path and considering them separately from the remaining $\log N-1$ (even) stages. From Fig. 3, we can see that in the worst scenario all connections passing through the middle stage on the tagged path are those from $I_{(\log N+1) / 2}$ and those destined for $O_{(\log N+1) / 2}$, and these two sets of connections share the same paths. Since $\left|I_{(\log N+1) / 2}\right|=\left|O_{(\log N+1) / 2}\right|=\sqrt{N / 2}$, and each of these connections may go to (come from) any of the $N-1$ output (input) ports, an multiplicative factor of $(r \times(\sqrt{(N / 2)} /(N-$ 1)) $)^{\sqrt{N / 2}}$ as the probability for these connections is applied to 


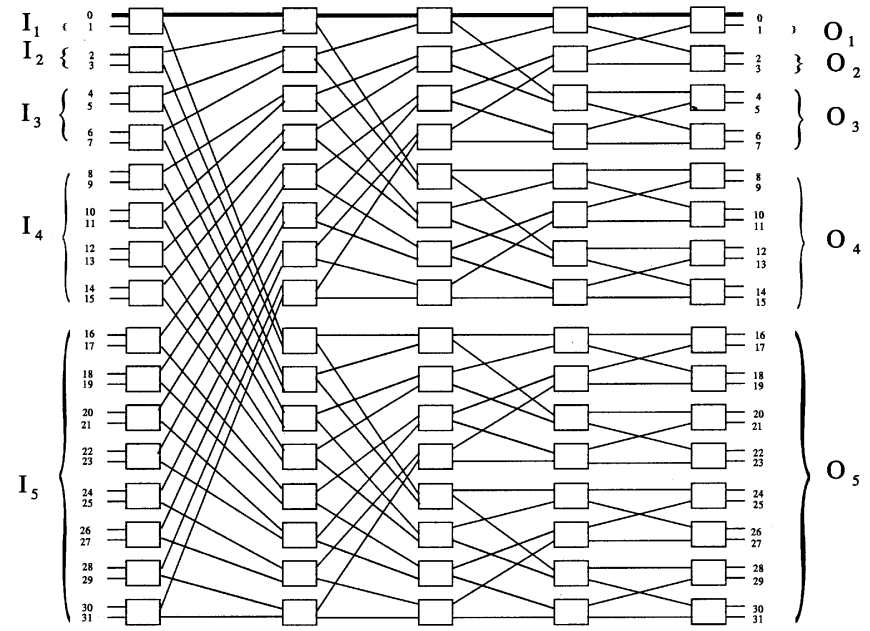

Fig. 3. A $32 \times 32$ banyan network (odd number of stages).

the probability defined by (2) for the remaining $\log N-1$ stages. This results in (3).

QED.

As a quantitative illustration, we evaluate (2) for $r=0.9$ and $N=64$ and (3) for $r=0.9$ and $N=128$, respectively, to obtain the equation shown at bottom of the page.

The above results indicate that the probability of the worst-case scenario is very small for both even and odd number of stages. So, spending a large amount of extra hardware in order to guarantee the strictly nonblocking property is not cost-effective in most cases. This motivates us to find out the blocking probability of a VSOB network with respect to the number of planes (hardware cost), and to seek an approach to making tradeoff between hardware cost and blocking probability. In Sections III-V, we will derive both the upper and lower bounds on blocking probability of a VSOB network to characterize the overall blocking behaviors of the network. We will show that the hardware cost required for a nonblocking VSOB network can be significantly reduced if a negligible nonzero blocking probability is allowed.

\section{UPPER BOUND ON BLOCKING PROBABILITY}

In this section, we shall develop the upper bound on blocking probability of VSOB $(N, m)$ for the cases of even and odd numbers of stages respectively. When $\log N$ is even, the tagged path is composed of two symmetric halves, each with $\log N / 2$ stages, where the second half mirrors the first half. The connections destined for output ports in the second half have the same properties as those originated from the input ports in the first half.
When $\log N$ is odd, we need to take out the middle stage and consider it separately in order to make the network symmetric as in the case when $\log N$ is even.

\section{A. Upper Bound on Blocking Probability When $\log N$ is Even}

Similar to the deterministic case analysis, under the constraint of crosstalk-free, the worst-case conflicting scenario for the tagged path is when all inputs in set $I_{i}$ are destined for the outputs in set $O_{(\log N-i+1)}$ and all outputs in set $O_{i}$ are originated from the inputs in set $I_{(\log N-i+1)}$ for $1 \leq i \leq(1 / 2) \log N$. Thus, the maximum number of conflicts with the tagged path is determined by both the connections originated from set $I_{\text {even }}$ and the connections destined for set $O_{\text {even }}$. Given the case there are $n_{I}$ connections from input set $I_{\text {even }}$ and $n_{O}$ connections destined for $O_{\text {even }}$ that block the tagged path, among which there are $k$ connections from $I_{\text {even }}$ that are destined for $O_{\text {even }}$. Under the "conservative" routing control strategy that each of these connections should be in a separate plane to guarantee the crosstalk nonblocking (and thus the link nonblocking), the maximum number of required planes will be $n_{I}+n_{O}-k$. Clearly there will be no blocking in $\operatorname{VSOB}(N, m)$ if $n_{I}+n_{O}-k<m$. Hereafter in the paper, we will use $\operatorname{Pr}(A)$ to denote that probability that event $A$ happens, $\operatorname{Pr}^{+}(A)$ and $\operatorname{Pr}^{-}(A)$ for the upper bound and lower bound of $\operatorname{Pr}(A)$ respectively. We shall establish the following theorem that gives an upper bound of blocking probability.

Theorem 2: For VSOB $(N, m)$, where $\log N$ is even, we have (6), shown at the bottom of the next page, with (7)-(9), also shown at the bottom of the next page. Here, $\alpha=r \times$ $(\sqrt{N}-1) /(N-1)$ is the probability that a connection from $I_{\text {even }}$ blocks the tagged path and is destined for $O_{\text {even }}$, and $\beta_{i}$ is the probability that a connection from $I_{i}$ blocks the tagged path but is not destined for $O_{\text {even }}$ as given in (5) ( $\beta_{i}$ is also the probability for a connection destined for $O_{i}$ blocking the tagged path but not originated from $\left.I_{\text {even }}\right), L_{i}$ and $T_{i}$ are the numbers of connections from $I_{i}$ (to $O_{i}$ ) blocking the tagged path, that are destined and not destined for (originated and not originated from) $O_{\text {even }}\left(I_{\text {even }}\right)$, respectively.

Proof: From $n_{I}+n_{O}-k<m, k \geq \max$ $\left\{0, n_{I}+n_{O}-m+1\right\}$. On the other hand, apparently $k \leq \min \left\{n_{1}, n_{0}\right\}$. Thus, given that there are $n_{I}$ connections from $I_{\text {even }}$ and $n_{O}$ connections destined for $O_{\text {even }}$ that all block the tagged path, we have

$$
\begin{aligned}
\operatorname{Pr}\left(n_{I}+n_{O}-k\right. & \left.\leq m-1 \mid n_{I}, n_{O}\right) \\
& =\sum_{k=\max \left\{0, n_{I}+n_{O}-m+1\right\}}^{\min \left\{n_{I}, n_{O}\right\}} \operatorname{Pr}\left(k \mid n_{I}, n_{O}\right) .
\end{aligned}
$$

$$
P_{\mathrm{worst}}=\left\{\begin{array}{l}
\left(\prod_{i=1}^{3}\left(0.9 \times \frac{\frac{64}{2^{i-1}-8}}{64-1}\right)^{2^{i-1}}\right)^{2} \approx 2.57 \times 10^{-10} \\
\left(\prod_{i=1}^{3}\left(0.9 \times \frac{\frac{128}{2^{i}-1}-16}{128-1}\right)^{2^{i-1}}\right)^{2} \cdot\left(0.9 \times \frac{8}{128-1}\right)^{8} \approx 2.45 \times 10^{-20}
\end{array}\right.
$$


Let $\operatorname{Pr}\left(n_{I}, n_{O}, k\right)$ be the probability of the event that there are $n_{I}$ connections from $I_{\text {even }}$ and $n_{O}$ connections destined for $O_{\text {even }}$ that block the tagged path, among which $k$ connections from $I_{\text {even }}$ are destined for $O_{\text {even }}$. Probabilities $\operatorname{Pr}\left(n_{I}, k\right)$, $\operatorname{Pr}\left(n_{O}, k\right), \operatorname{Pr}\left(n_{I}, n_{O}\right)$ and $\operatorname{Pr}(k)$ are also defined in the same way as for $\operatorname{Pr}\left(n_{I}, n_{O}, k\right)$. Following the assumption that the statuses of individual input (output) ports in the network are independent, we have

$$
\begin{aligned}
\operatorname{Pr}\left(n_{I}, n_{O}\right) \cdot \operatorname{Pr}\left(k \mid n_{O}, n_{I}\right) & =\operatorname{Pr}\left(k, n_{I}, n_{O}\right) \\
& =\operatorname{Pr}\left(n_{I}, k\right) \cdot \operatorname{Pr}\left(n_{O} \mid n_{I}, k\right) \\
& =\operatorname{Pr}\left(n_{I}, k\right) \cdot \operatorname{Pr}\left(n_{O} \mid k\right) \\
& =\frac{\operatorname{Pr}\left(n_{I}, k\right) \cdot\left(n_{O}, k\right)}{\operatorname{Pr}(k)}
\end{aligned}
$$

Therefore, for $\operatorname{VSOB}(N, m)$ when $\log N$ is even, from (10) and (11) the probability $\operatorname{Pr}$ (nonblocking) is then given by the equation at the bottom of the page. From the meanings of $\alpha, \beta_{i}$, $L_{i}$ and $T_{i}$, (7)-(9) on $\operatorname{Pr}(k), \operatorname{Pr}\left(n_{I}, k\right)$ and $\operatorname{Pr}\left(n_{O}, k\right)$ can be easily established. Note that $\left|I_{\text {even }}\right|=\left|O_{\text {even }}\right|=\sqrt{N-1}$ and $\alpha+\beta_{i}$ is the probability that the connections from $I_{i}$ (for $O_{i}$ ) block the tagged path regardless whether they are terminated at $O_{\text {even }}$ (from $I_{\text {even }}$ ) or not.

QED
The following corollary shows that the upper bound blocking probability derived above matches the deterministic condition for strictly nonblocking.

Corollary 1: When $\log N$ is even, the blocking probability $\mathrm{Pr}^{+}$(blocking) given in (6) becomes 0 if $m \geq 2 \sqrt{N}-1$.

Proof: Since $n_{I} \leq\left|I_{\text {even }}\right|=\sum_{i=1}^{(1 / 2) \log N}\left|I_{i}\right|=\sqrt{N}-1$, $n_{O} \leq\left|O_{\text {even }}\right|=\sum_{i=1}^{(1 / 2)} \log N\left|O_{i}\right|=\sqrt{N}-1, k \geq 0$, and the events $n_{I}=\sqrt{N}-1, k=0$ and $n_{O}=\sqrt{N}-1$ are compatible, then we have

$$
n_{I}+n_{O}-k \leq 2(\sqrt{N}-1) .
$$

Thus, for any given $n_{I}$ and $n_{O}$, if $m \geq 2(\sqrt{N}-1)+1=$ $2 \sqrt{N}-1$ we always have

$$
\operatorname{Pr}\left(n_{I}+n_{O}-k \geq m \mid n_{I}, n_{O}\right)=0 .
$$

Therefore, when $m \geq 2 \sqrt{N}-1$, the probability that the connection is blocked is given by

$$
\begin{aligned}
\operatorname{Pr}^{+}(\text {blocking })= & \sum_{n_{I}=0}^{\min \{m-1, \sqrt{N}-1\}} \sum_{n_{O}=0}^{\min \{m-1, \sqrt{N}-1\}} \\
& \times \operatorname{Pr}\left(n_{I}+n_{o}-k \geq m \mid n_{I}, n_{o}\right) \\
& \cdot \operatorname{Pr}\left(n_{I}, n_{o}\right)=0 . \quad \text { QED }
\end{aligned}
$$

$$
\begin{aligned}
\operatorname{Pr}^{+}(\text {blocking }) & =1-\sum_{n_{I}=0}^{\min \{m-1, \sqrt{N}-1\}} \sum_{n_{O}=0}^{\min \{m-1, \sqrt{N}-1\}} \operatorname{Pr}\left(n_{I}+n_{O}-k<m \mid n_{I}, n_{O}\right) \cdot \operatorname{Pr}\left(n_{I}, n_{o}\right) \\
& =1-\sum_{n_{I}=0}^{\min \{m-1, \sqrt{N}-1\}} \sum_{n_{O}=0}^{\min \{m-1, \sqrt{N}-1\}} \sum_{k=\max \left\{0, n_{I}+n_{O}-m+1\right\}}^{\min \left\{n_{I}, n_{O}\right\}} \frac{\operatorname{Pr}\left(n_{I}, k\right) \cdot \operatorname{Pr}\left(n_{O}, k\right)}{\operatorname{Pr}(k)}
\end{aligned}
$$

$$
\begin{aligned}
& \operatorname{Pr}(k)=\left(\begin{array}{c}
\sqrt{N}-1 \\
k
\end{array}\right) \cdot \alpha^{k} \cdot(1-\alpha)^{\sqrt{N}-1-k} \\
& \operatorname{Pr}\left(n_{I}, k\right)
\end{aligned}
$$

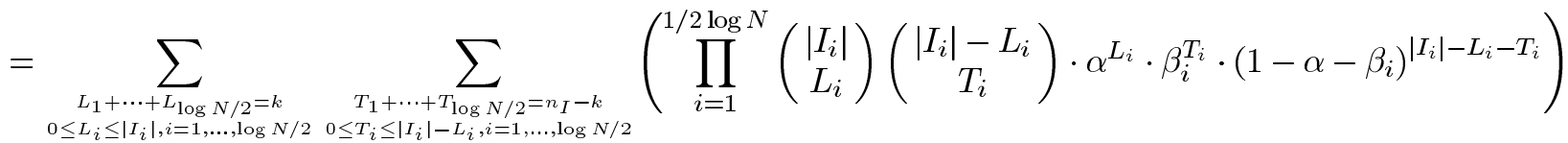

$$
\begin{aligned}
& \operatorname{Pr}\left(n_{O}, k\right) \\
& =\sum_{\substack{L_{1}+\ldots+L_{\log } N / 2=k \\
0 \leq L_{i} \leq\left|O_{i}\right|, i=1, \ldots, \log N / 2}} \sum_{\substack{T_{1}+\cdots+T_{\log N / 2}=n_{0}-k \\
0 \leq T_{i} \leq\left|O_{i}\right|-L_{i}, i=1, \ldots, \log N / 2}}\left(\prod_{i=1}^{1 / 2 \log N}\left(\begin{array}{c}
\left|O_{i}\right| \\
L_{i}
\end{array}\right)\left(\begin{array}{c}
\left|O_{i}\right|-L_{i} \\
T_{i}
\end{array}\right) \cdot \alpha^{L_{i}} \cdot \beta_{i}^{T_{i}} \cdot\left(1-\alpha-\beta_{i}\right)^{\left|O_{i}\right|-L_{i}-T_{i}}\right.
\end{aligned}
$$

$$
\begin{aligned}
\operatorname{Pr}(\text { nonblocking }) & =\sum_{n_{I}=0}^{\min \left\{m-1,\left|I_{\text {even }}\right|\right\}} \sum_{n_{O}=0}^{\min \left\{m-1,\left|O_{\text {even }}\right|\right\}} \operatorname{Pr}\left(n_{I}+n_{O}-k \leq m-1 \mid n_{I}, n_{O}\right) \cdot \operatorname{Pr}\left(n_{I}, n_{O}\right) \\
& =\sum_{n_{I}=0}^{\min \{m-1, \sqrt{N}-1\}} \sum_{n_{O}=0}^{\min \{m-1, \sqrt{N}-1\}} \sum_{k=\max \left\{0, n_{I}+n_{O}-m+1\right\}}^{\min \left\{n_{I}, n_{O}\right\}} \frac{\operatorname{Pr}\left(n_{I}, k\right) \cdot \operatorname{Pr}\left(n_{O}, k\right)}{\operatorname{Pr}(k)}
\end{aligned}
$$


Since the "conservative" routing control strategy, in which each of these connections that block the tagged path should be in a separate plane, has been used in developing the (6), the blocking probability of a connection request under any routing control strategy is upper bounded by the blocking probability given in (6).

\section{B. Upper Bound on Blocking Probability When $\log N$ is Odd}

For the case when $\log N$ is odd (Fig. 3), we define input set $I_{\text {odd }}$ and output set $O_{\text {odd }}$ as:

$$
I_{\text {odd }}=\bigcup_{i=1}^{(\log N+1) / 2} I_{i}, \quad O_{\text {odd }}=\bigcup_{i=1}^{(\log N+1) / 2} O_{i} .
$$

Under the constraint of crosstalk-free, the worst-case scenario of conflicting for the tagged path is when all inputs in set $I_{i}$ are destined for the outputs in set $O_{(\log N-i+1)}$ and all outputs in set $O_{i}$ are originated from the inputs in set $I_{(\log N-i+1)}$ for $1 \leq$ $i \leq(1 / 2)(\log N+1)$. Thus, the maximum number of conflicts with the tagged path is determined by both the connections from set $I_{\text {odd }}$ and the connections destined for set $O_{\text {odd }}$.

Following a similarly discussion to that for the case when $\log N$ is even and replacing $I_{\text {even }}$ and $O_{\text {even }}$ with $I_{\text {odd }}$ and $O_{\text {odd }}$, respectively, we can obtain the upper-bound blocking probability for a $\operatorname{VSOB}(N, m)$ when $\log N$ is odd.

Theorem 3: For a VSOB $(N, m)$, where $\log N$ is odd, a upper bound of blocking probability is given by

$$
\begin{aligned}
\operatorname{Pr} & + \text { (blocking) } \\
= & 1-\sum_{n_{I}=0}^{\min \{m-1, \sqrt{2 N}-1\}} \sum_{n_{O}=0}^{\min \{m-1, \sqrt{2 N}-1\}} \operatorname{Pr} \\
& \times\left(n_{I}+n_{O}-k<m\right. \\
=1-\sum_{\left.n_{I}, n_{O}\right) \cdot \operatorname{Pr}\left(n_{I}, n_{O}\right)}^{\min \{m-1, \sqrt{2 N}-1\}} & \sum_{n_{I}=0}^{\min \{m-1, \sqrt{2 N}-1\}} \\
& \sum_{k=\max \left\{0, n_{I}+n_{O}-m+1\right\}}^{\min \left\{n_{I}, n_{O}\right\}} \frac{\operatorname{Pr}\left(n_{I}, k\right) \cdot \operatorname{Pr}\left(n_{O}, k\right)}{\operatorname{Pr}(k)}
\end{aligned}
$$

where

$$
\begin{aligned}
& \operatorname{Pr}(k)=\left(\begin{array}{c}
\sqrt{2 N}-1 \\
k
\end{array}\right) \cdot \alpha^{k} \cdot(1-\alpha)^{\sqrt{2 N}-1-k} \\
& \operatorname{Pr}\left(n_{I}, k\right)=\sum_{\substack{L_{1}+\cdots+L_{(\log N+1) / 2}=k \\
0 \leq L_{i} \leq\left|I_{i}\right|, i=1, \ldots,(\log N+1) / 2}} \\
& \sum_{\substack{T_{1}+\cdots+T_{(\log N-1) / 2}=n_{I}-k \\
0 \leq T_{i} \leq\left|I_{i}\right|-L_{i}, i=1, \ldots,(\log N-1)}} \\
& \left(\prod_{i=1}^{(\log N-1) / 2}\left(\begin{array}{c}
\left|I_{i}\right| \\
L_{i}
\end{array}\right)\left(\begin{array}{c}
\left|I_{i}\right|-L_{i} \\
T_{i}
\end{array}\right)\right. \\
& \left.\cdot \alpha^{L_{i}} \cdot \beta_{i}^{T_{i}} \cdot\left(1-\alpha-\beta_{i}\right)^{\left|I_{i}\right|-L_{i}-T_{i}}\right) \\
& \times \operatorname{Pr}^{*}
\end{aligned}
$$

$$
\begin{aligned}
& \operatorname{Pr}\left(n_{O}, k\right)=\sum_{\substack{L_{1}+\cdots+L_{(\log N+1) / 2}=k \\
0 \leq L_{i} \leq\left|O_{i}\right|, i=1, \ldots,(\log N+1) / 2}} \\
& \sum_{T_{1}+\cdots+T_{(\log N-1) / 2}=n_{O}-k} \\
& 0 \leq T_{i} \leq\left|O_{i}\right|-L_{i}, i=1, \ldots,(\log N-1) / 2 \\
& \left(\prod_{i=1}^{(\log N-1) / 2}\left(\begin{array}{c}
\left|O_{i}\right| \\
L_{i}
\end{array}\right)\left(\begin{array}{c}
\left|O_{i}\right|-L_{i} \\
T_{i}
\end{array}\right)\right. \\
& \left.\cdot \alpha^{L_{i}} \cdot \beta_{i}^{T_{i}} \cdot\left(1-\alpha-\beta_{i}\right)^{\left|O_{i}\right|-L_{i}-T_{i}}\right) \\
& \times \operatorname{Pr}^{*} \\
& \operatorname{Pr}^{*}=\left(\begin{array}{c}
\left|I_{(\log N+1) / 2}\right| \\
L_{(\log N+1) / 2}
\end{array}\right) \cdot \alpha^{L_{(\log N+1) / 2}} \\
& \cdot(1-\alpha)^{\left|I_{(\log N+1) / 2}\right|-L_{(\log N+1) / 2}} \\
& \alpha=r \cdot \frac{\sqrt{2 N}-1}{N-1}, \beta_{i}=r \cdot \frac{N / 2^{i-1}-\sqrt{2 N}}{N-1}, \\
& i=1, \ldots, \frac{(\log N-1)}{2} \text {. }
\end{aligned}
$$

The proof is the same as for Theorem 2, with the exception of separating treatment for the middle stage which brought the extra probability factor $\mathrm{Pr}^{*}$.

The following corollary shows that, when $\log N$ is odd, the upper bound blocking probability also agrees with the deterministic condition for strictly nonblocking.

Corollary 2: When $\log N$ is odd, the blocking probability $\mathrm{Pr}^{+}$(blocking) given in (12) becomes 0 if $m \geq(3 / 2) \sqrt{2 N}-1$.

Proof: The proof for Corollary 2 is the same as that for Corollary 1, except that when the $\log N$ is odd, the worst-case scenario is when all inputs in set $I_{i}$ are destined for the outputs in set $O_{(\log N-i+1)}$ and all outputs in $O_{i}$ are originated from set $I_{(\log N-i+1)}$ for $1 \leq i \leq(1 / 2)(\log N+1)$. Note that all the inputs in set $I_{(1 / 2)(\log N+1)}$ are destined for the outputs in set $O_{(1 / 2)(\log N+1)}$ and these connections share the same paths. Thus, only half of the total elements in these two sets can be counted, and this gives

$$
\begin{array}{r}
n_{I}+n_{O}-k \leq\left|I_{\text {odd }}\right|+\left|O_{\text {odd }}\right|-\left|I_{(1 / 2)(\log N+1)}\right| \\
=\left(\frac{3}{2}\right) \sqrt{2 N}-2 .
\end{array}
$$

QED.

\section{LOWER BOUND ON BLOCKING PROBABILITY}

Similar to the analysis of upper-bound blocking probability, in this Section we will develop the lower-bound probability for the cases of even and odd numbers of stages respectively. Let $n_{i}$ be the number of connections passing through the $i$ th tagged SE, and $n_{\max }=\max _{i}\left\{n_{i}\right\}$. Under the constraint of crosstalkfree, the minimum number of required planes will be determined by the $n_{\max }$. For VSOB $(N, m)$, let $\operatorname{Pr}\left(n_{\max }<m\right)$ be the nonblocking probability. A lower-bound blocking probability is thus

$$
\operatorname{Pr}^{-}(\text {Blocking })=1-\operatorname{Pr}\left(n_{\max }<m\right) .
$$




\section{A. Lower-Bound Blocking Probability When $\log N$ is Even}

From (18), we need to first evaluate the probability $\operatorname{Pr}\left(n_{\max }<m\right)$ to get the lower-bound blocking probability. By the definition of $n_{\max }$, we have

$$
\begin{aligned}
\operatorname{Pr}\left(n_{\max }<m\right)= & \operatorname{Pr}\left(n_{1}<m, \ldots, n_{\log N}<m\right) \\
= & \operatorname{Pr}\left(n_{1}<m, \ldots, n_{(1 / 2) \log N}<m\right) \\
& \cdot \operatorname{Pr}\left(n_{(1 / 2) \log N+1}<m, \ldots, n_{\log N}<m\right. \\
& \left.\mid n_{1}<m, \ldots, n_{(1 / 2) \log N}<m\right) .
\end{aligned}
$$

For the tagged path, the connections passing through the $(i+1)$ th tagged SE will be only determined by the connections coming from the $i$ th tagged SE and from input intersecting set $I_{i+1}$. So

$\operatorname{Pr}\left(n_{(1 / 2) \log N+1}<m, \ldots, n_{\log N}<m \mid n_{1}\right.$

$$
\left.<m, \ldots, n_{(1 / 2) \log N}<m\right)
$$

$=\operatorname{Pr}\left(n_{(1 / 2) \log N+1}<m, \ldots, n_{\log N}<m \mid n_{(1 / 2) \log N}<m\right)$

$=\operatorname{Pr}\left(n_{(1 / 2)} \log N+1<m \mid n_{(1 / 2)} \log N<m\right)$

$\cdot \operatorname{Pr}\left(n_{(1 / 2) \log N+2}<m, \ldots, n_{\log N}<m \mid n_{(1 / 2) \log N+1}<m\right)$

$=\operatorname{Pr}\left(n_{(1 / 2) \log N+1}<m \mid n_{(1 / 2)} \log N<m\right)$

$$
\cdot \frac{\operatorname{Pr}\left(n_{(1 / 2) \log N+1}<m, \ldots, n_{\log N}<m\right)}{\operatorname{Pr}\left(n_{(1 / 2)} \log N+1<m\right)} .
$$

The following expression can then be obtained using the symmetry of the network when $\log N$ is even:

$$
\begin{aligned}
\operatorname{Pr}\left(n_{\max }<m\right)= & \operatorname{Pr}\left(n_{1}<m, \ldots, n_{\log N}<m\right) \\
= & \operatorname{Pr}\left(n_{1}<m, \ldots, n_{(1 / 2) \log N}<m\right) \\
& \cdot \operatorname{Pr}\left(n_{(1 / 2) \log N+1}<m, \ldots, n_{\log N}<m\right) \\
& \cdot \frac{\operatorname{Pr}\left(n_{(1 / 2) \log N+1}<m \mid n_{(1 / 2) \log N}<m\right)}{\operatorname{Pr}\left(n_{(1 / 2) \log N+1}<m\right)} \\
= & \left(\operatorname{Pr}\left(n_{1}<m, \ldots, n_{(1 / 2) \log N}<m\right)\right)^{2} \\
& \cdot \frac{\operatorname{Pr}\left(n_{(1 / 2) \log N}<m, n_{(1 / 2) \log N+1}<m\right)}{\left(\operatorname{Pr}\left(n_{(1 / 2)} \log N<m\right)\right)^{2}} .
\end{aligned}
$$

Since

$$
\begin{aligned}
& \operatorname{Pr}\left(n_{1}<m, \ldots, n_{(1 / 2) \log N}<m\right)=\operatorname{Pr}\left(n_{1}<m\right) \\
& \quad \cdot \operatorname{Pr}\left(n_{2}<m, \ldots, n_{(1 / 2) \log N}<m \mid n_{1}<m\right) \\
& =\operatorname{Pr}\left(n_{1}<m\right) \cdot \operatorname{Pr}\left(n_{2}<m \mid n_{1}<m\right) \\
& \quad \cdot \operatorname{Pr}\left(n_{3}<m, \ldots, n_{(1 / 2) \log N}<m \mid n_{2}<m\right)
\end{aligned}
$$

applying the process above recursively, we have

$$
\begin{aligned}
& \operatorname{Pr}\left(n_{1}<m, \ldots, n_{(1 / 2) \log N}<m\right)=\operatorname{Pr}\left(n_{1}<m\right) \\
& \quad \cdot \prod_{i=1}^{(1 / 2) \log N-1} \operatorname{Pr}\left(n_{i+1}<m \mid n_{i}<m\right) \\
& =\operatorname{Pr}\left(n_{1}<m\right) \\
& \quad \frac{\prod_{i=1}^{(1 / 2) \log N-1} \operatorname{Pr}\left(n_{i}<m, n_{i+1}<m\right)}{\operatorname{Pr}\left(n_{i}<m\right)} .
\end{aligned}
$$

Thus, $\operatorname{Pr}\left(n_{\max }<m\right)$ is given by

$$
\begin{aligned}
& \operatorname{Pr}\left(n_{\max }<m\right) \\
& =\left(\operatorname{Pr}\left(n_{1}<m\right) \cdot \prod_{i=1}^{(1 / 2) \log N-1} \frac{\operatorname{Pr}\left(n_{i}<m, n_{i+1}<m\right)}{\operatorname{Pr}\left(n_{i}<m\right)}\right)^{2} \\
& \quad \cdot \frac{\operatorname{Pr}\left(n_{(1 / 2) \log N}<m, n_{(1 / 2) \log N+1}<m\right)}{\left(\operatorname{Pr}\left(n_{(1 / 2) \log N}<m\right)\right)^{2}} .
\end{aligned}
$$

To calculate $\operatorname{Pr}\left(n_{i}<m, n_{i+1}<m\right)$ for $i=1,2, \ldots,(1 / 2) \log N, \operatorname{Pr}\left(n_{i}<m, n_{i+1}<m\right)$ is first expressed as

$$
\begin{aligned}
& \operatorname{Pr}\left(n_{i}<m, n_{i+1}<m\right) \\
& =\sum_{k=0}^{\min \left\{m-1, \sum_{j=1}^{i}\left|I_{j}\right|, \sum_{j=1}^{\log N-i+1}\left|O_{j}\right|\right\}} \\
& \quad \times \sum_{l=0}^{\min \left\{m-1,\left|I_{i+1}\right|+k, \sum_{j=1}^{\log N-i}\left|O_{j}\right|\right\}} \\
& \times \operatorname{Pr}\left(n_{i}=k, n_{i+1}=l\right) \\
& =\sum_{k=0}^{\min \left\{m-1, \sum_{j=1}^{i}\left|I_{j}\right|, \sum_{j=1}^{\log N-i+1}\left|O_{j}\right|\right\}} \\
& \quad \min \left\{m-1,\left|I_{i+1}\right|+k, \sum_{j=1}^{\log N-i}\left|O_{j}\right|\right\} \\
& \sum_{l=0}^{\min \{k, l\}} \operatorname{Pr}\left(n_{i}=k, n_{i+1}=l, t_{i, i+1}=c\right)
\end{aligned}
$$

where $t_{i, i+1}$ is the number of connections passing through both the $i$ th and the $(i+1)$ th tagged SEs. Since the correlation between $n_{i}$ and $n_{i+1}$ is determined by the connections passing through both the $i$ th and the $(i+1)$ th tagged SEs, we have

$$
\begin{aligned}
\operatorname{Pr}\left(n_{i}=k, n_{i+1}=\right. & \left.l, t_{i, i+1}=c\right) \\
= & \operatorname{Pr}\left(n_{i}=k, t_{i, i+1}=c\right) \\
& \cdot \operatorname{Pr}\left(n_{i+1}=l \mid n_{i}=k, t_{i, i+1}=c\right) \\
= & \operatorname{Pr}\left(n_{i}=k, t_{i, i+1}=c\right) \\
& \cdot \operatorname{Pr}\left(n_{i+1}=l \mid t_{i, i+1}=c\right) .
\end{aligned}
$$

For $i=1,2, \ldots,(1 / 2) \log N, \operatorname{Pr}\left(n_{i}=k, t_{i, i+1}=c\right)$ is given by

$$
\begin{aligned}
& \operatorname{Pr}\left(n_{i}=k, t_{i, i+1}=\right.c)=\left(\begin{array}{c}
\sum_{j=1}^{i}\left|I_{i}\right| \\
c
\end{array}\right)\left(\begin{array}{c}
\sum_{j=1}^{i}\left|I_{i}\right| \\
k-c
\end{array}\right) \\
& \cdot \eta_{i}^{c} \cdot \xi_{i}^{k-c} \cdot\left(1-\eta_{i}-\xi_{i}\right)^{\sum_{j=1}^{i}\left|I_{i}\right|-k} .
\end{aligned}
$$

Here $\eta_{i}$ is the probability that a connection from $I_{1}, \ldots, I_{i}$ will pass through both the $i$ th and the $(i+1)$ th tagged SEs, and $\xi_{i}$ is the probability that a connection from $I_{1}, \ldots I_{i}$ will pass 
through the $i$ th tagged SE but not the $(i+1)$ th tagged SE. $\eta_{i}$ and $\xi_{i}$ are given by

$$
\eta_{i}=r \cdot\left(\frac{\frac{N}{2^{i}}-1}{N-1}\right), \quad \xi_{i}=r \cdot\left(\frac{\frac{N}{2^{i}}}{N-1}\right) .
$$

For $i=1,2, \ldots,(1 / 2) \log N-1$, the conditional probability $\operatorname{Pr}\left(n_{i+1}=l \mid t_{i, i+1}=c\right)$ is evaluated by

$$
\begin{aligned}
\operatorname{Pr}\left(n_{i+1}=l \mid\right. & \left.t_{i, i+1}=c\right) \\
& =\left(\begin{array}{c}
\left|I_{i+1}\right| \\
l-c
\end{array}\right) \cdot \mu_{i+1}^{l-c} \cdot\left(1-\mu_{i+1}\right)^{\left|I_{i+1}\right|-l+c}
\end{aligned}
$$

where $\mu_{i+1}$ is the probability that a connection from $I_{i+1}$ will pass through the $(i+1)$ th tagged SE under the condition that there are $c$ connections from $I_{1}, \ldots, I_{i}$ have passed through the $(i+1)$ th tagged SE. $\mu_{i+1}$ is given by

$$
\mu_{i+1}=r \cdot \frac{\left(\frac{N}{2^{i}}-1-c\right)}{(N-1-c)}
$$

When $i=(1 / 2) \log N$, the maximum number of possible connections is limited by the number of output ports available rather than the number of input ports, so the conditional probability $\operatorname{Pr}\left(n_{i+1}=l \mid t_{i, i+1}=c\right)$ will be determined by

$$
\begin{aligned}
\operatorname{Pr} & \left(n_{(1 / 2) \log N+1}=l \mid t_{(1 / 2) \log N,(1 / 2) \log N+1}=c\right) \\
= & \left(\begin{array}{c}
\sum_{j=1}^{(1 / 2) \log N}\left|O_{j}\right|-c \\
l-c
\end{array}\right) \\
& \cdot \lambda^{l-c} \cdot(1-\lambda)^{\sum_{j=1}^{(1 / 2) \log N}\left|O_{j}\right|-l}
\end{aligned}
$$

where $\lambda$ is the probability that a connection destined for $O_{\text {even }}$ comes from under the condition that there are $c$ connections from $I_{\text {even }}$ have passed through the $((1 / 2) \log N+1)$ th tagged $\mathrm{SE}$, which is evaluated as:

$$
\lambda=r \cdot \frac{\left|I_{(1 / 2) \log N+1}\right|}{N-1-\sum_{j=1}^{(1 / 2) \log N}\left|I_{j}\right|} .
$$

For $i=1,2, \ldots,(1 / 2) \log N, \operatorname{Pr}\left(n_{i}<m\right)$ can be evaluated using the following expression:

$$
\begin{aligned}
\operatorname{Pr}\left(n_{i}<m\right)= & \sum_{k=0}^{\min \left\{m-1, \sum_{j=1}^{i}\left|I_{j}\right|\right\}} \operatorname{Pr}\left(n_{i}=k\right) \\
= & \sum_{k=0}^{\min \left\{m-1, \sum_{j=1}^{i}\left|I_{j}\right|\right\}}\left(\begin{array}{c}
\sum_{j=1}^{i}\left|I_{j}\right| \\
k
\end{array}\right) \\
& \cdot\left(\eta_{i}+\xi_{i}\right)^{k}\left(1-\eta_{i}-\xi_{i}\right)^{\sum_{j=1}^{i}\left|I_{j}\right|-k} .
\end{aligned}
$$

Summarizing the above derivation, we have the following theorem.
Theorem 4: For VSOB $\log N$, when $\log N$ is even, a lower bound of blocking probability is

$$
\begin{aligned}
\operatorname{Pr}^{-}(\text {blocking })= & 1-\operatorname{Pr}\left(n_{\max }<m\right) \\
= & 1-\left(\operatorname{Pr}\left(n_{1}<m\right) \cdot \prod_{i=1}^{(1 / 2) \log N-1}\right. \\
& \left.\times \frac{\operatorname{Pr}\left(n_{i}<m, n_{i+1}<m\right)}{\operatorname{Pr}\left(n_{i}<m\right)}\right)^{2} \\
& \cdot \frac{\operatorname{Pr}\left(n_{(1 / 2) \log N}<m, n_{(1 / 2) \log N+1}<m\right)}{\left(\operatorname{Pr}\left(n_{(1 / 2) \log N}<m\right)\right)^{2}}
\end{aligned}
$$

where $\operatorname{Pr}\left(n_{i}<m\right)$ (including $\operatorname{Pr}\left(n_{1}<m\right)$ and $\left.\operatorname{Pr}\left(n_{(1 / 2) \log N}<m\right)\right)$ and $\operatorname{Pr}\left(n_{i}<m, n_{i+1}<m\right)$ (including $\left.\operatorname{Pr}\left(n_{(1 / 2) \log N}<m, n_{(1 / 2)} \log N+1<m\right)\right)$ are given by (28) and (20), respectively.

When the above lower bound on blocking probability approaches to zero, the above lower bound yields immediately the deterministic condition for the rearrangeably nonblocking $\operatorname{VSOB}(N, m)$ [7], [11]. This is given by the following corollary.

Corollary 3: When $\log N$ is even, the probability that a connection request is blocked, $\mathrm{Pr}^{-}$(blocking ) in (29), becomes 0 if $m \geq \sqrt{N}$

Proof: Note that the maximum number of possible connections passing through the $i$ th tagged $\mathrm{SE}(i=$ $1,2, \ldots, \log N)$ will be $\min \left\{\sum_{j=1}^{i}\left|I_{j}\right|, \sum_{j=1}^{\log N-i+1}\left|O_{j}\right|\right\}$. For the case when $\log N$ is even, we always have

$$
\begin{aligned}
n_{\max } & =\max _{i}\left\{n_{i}\right\} \\
& \leq \max _{i}\left\{\min \left(\sum_{j=1}^{i}\left|I_{j}\right|, \sum_{j=1}^{\log N-i+1}\left|O_{j}\right|\right)\right\}=\sqrt{N}-1 .
\end{aligned}
$$

This finishes the proof.

\section{B. Lower-Bound Blocking Probability When $\log N$ Is Odd}

Following the similar arguments as for the case when $\log N$ is even, a lower-bound blocking probability for $\operatorname{VSOB}(N, m)$ when $\log N$ is odd can also be derived:

Theorem 5: When $\log N$ is odd, a lower-bound blocking probability for $\operatorname{VSOB}(N, m)$ is

$$
\begin{aligned}
& \left.\operatorname{Pr}^{-} \text {(blocking }\right)=1-\operatorname{Pr}\left(n_{\max }<m\right) \\
& \quad=1-\frac{\left(\operatorname{Pr}\left(n_{1}<m\right) \cdot \prod_{i=1}^{(1 / 2)(\log N-1)} \frac{\operatorname{Pr}\left(n_{i}<m, n_{i+1}<m\right)}{\operatorname{Pr}\left(n_{i}<m\right)}\right)^{2}}{\operatorname{Pr}\left(n_{(1 / 2)(\log N+1)}<m\right)}
\end{aligned}
$$

where $\operatorname{Pr}\left(n_{i}<m, n_{i+1}<m\right)$ and $\operatorname{Pr}\left(n_{i}<m\right)$ can be evaluated using (20) and (28), respectively.

The following corollary shows that when $\log N$ is odd, the above lower-bound blocking probability also agrees with the deterministic condition for the rearrangeably nonblocking VSOB $(N, m)$ [7], [11]. 
Corollary 4: When $\log N$ is odd, the probability that a connection request is blocked, $\mathrm{Pr}^{-}$(blocking) in (30), becomes 0 if $m \geq \sqrt{2 N}$

The proof follows the same way as for Corollary 3 .

\section{EXPERIMENTAL RESULTS}

An extensive experimental study has been performed to validate our upper and lower bounds on blocking probability of a VSOB network. The network simulator we developed consists of two major modules: the request pattern generator and the request router. The request pattern generator randomly generates a connection request pattern for a VSOB network. The request router attempts to route the connection requests of the request pattern through the network using different routing strategies. Here the random routing and packing strategy are considered. Packing strategy is a commonly used routing control strategy for MINs [1], [2], [4], and it is generally believed that packing can improve network performance and reduce network cost. Under the packing strategy for a VSOB network, a connection is realized on a path found by trying the most used plane of the network first and least used plane last [7]. In the random routing, request router randomly chooses one of the planes that can be used by a request. If no plane can satisfy the request of tagged path using a routing strategy, the connection request pattern is recorded as a blocked connection pattern corresponding to the routing strategy. To verify further our upper bound and lower bound on blocking probability, we also use a "upper" routing strategy and a "lower" routing strategy to route the connection requests. In the "upper" routing, request router guarantees that each of these requests that block the tagged path should be in a separate plane. On the other hand, the "lower" routing strategy only guarantees that each of these requests that block a tagged $\mathrm{SE}$ will be in a distinct plane. If no plane can satisfy the request of tagged path using the "upper" ("lower") routing strategy, the connection request pattern is recorded as a blocked connection pattern corresponding to the upper bound (lower bound). The blocking probability of a routing strategy is then estimated by the ratio of number of blocked connection patterns for the strategy to the total number of connection patterns generated. During the simulation, a certain workload is maintained. The workload is measured by the network utilization, which is defined as the probability that an input (output) link is busy.

\section{A. Theoretical Versus Simulated Bounds on Blocking Probability}

We have examined two network configurations, $N=16$ and $N=32$, respectively. For each configuration, the blocking probabilities were generated by both theoretical bounds and simulator for $r=80 \%$ and $r=100 \%$. The comparisons results for $N=16$ and $N=32$ are summarized in Figs. 4 and 5. The results in Figs. 4 and 5 show that our theoretical bounds are correct in estimating both the upper- and lower-bound blocking probabilities of VSOB networks, and blocking probability of random routing is nicely bounded by our lower and upper bounds. It is interesting to note that the blocking probability of packing strategy matches nicely with the lower-bound blocking probability.

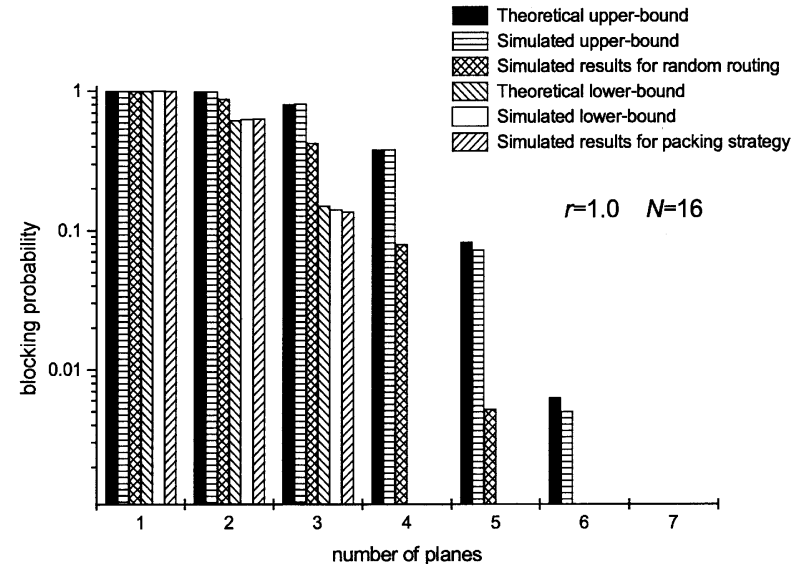

(a)

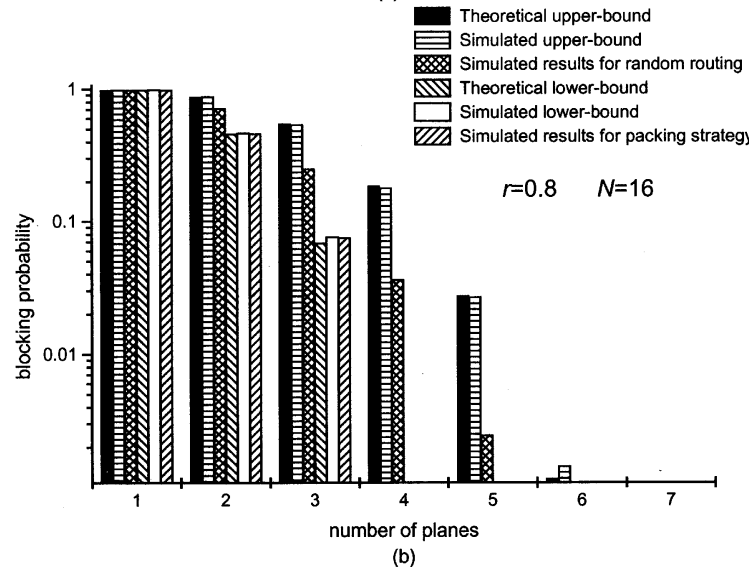

(b)

Fig. 4. Blocking probability of $16 \times 16$ VSOB network.

We should also see the theoretical data for upper bound follow closely with the condition of strictly nonblocking as shown in [13], and the theoretical data for lower bound follow closely with the condition of rearrangeably nonblocking under packing strategy as given in [7]. For $N=16$, the upper-bound blocking probability goes to zero at $m=2 \sqrt{N}-1=7$ and the lowerbound blocking probability goes to zero at $m=\sqrt{N}=4$. For $N=32$, the upper-bound blocking probability goes to zero at $m=(3 / 2) \sqrt{2 N}-1=11$ and the lower-bound blocking probability goes to zero at. $m=\sqrt{2 N}=8$.

The above results show that our bounds can accurately describe the blocking behaviors of VSOB networks. Our lower bound is useful in estimating the minimum blocking probability of a VSOB network and also the blocking probability of the network under packing strategy. The upper bound, however, can guide the network designers to estimate the maximum blocking probability of a VSOB network in which any routing strategy might be applied. Figs. 4 and 5 have shown clearly that it is possible for us to drastically reduce the hardware cost (number of planes) with only a little sacrifice in blocking probability of a network. So our bounds can be used to display the tradeoffs between hardware cost and blocking probability.

\section{B. Hardware Cost Versus Blocking Probability}

When the blocking probability (denoted by $B P$ thereafter) is required to be less than $1 \%$ for VSOB networks of different sizes, the minimum numbers of planes estimated by our upper bound are summarized in Fig. 6 for different workloads $(r)$. 


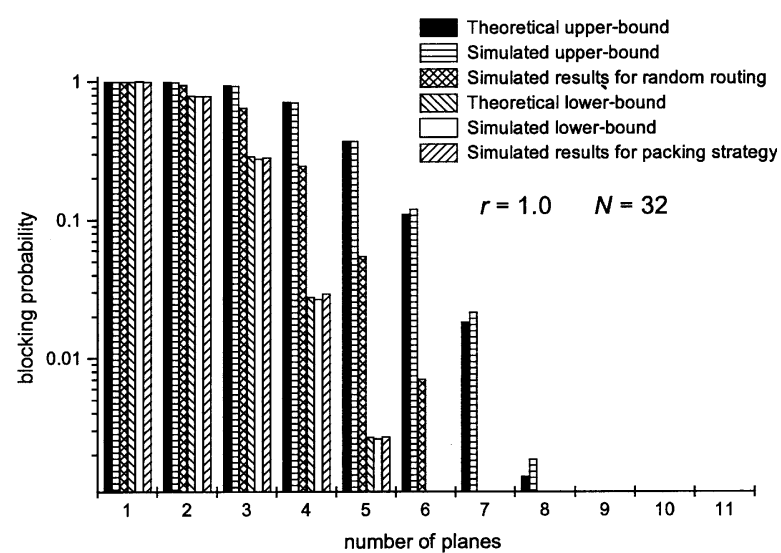

(a)

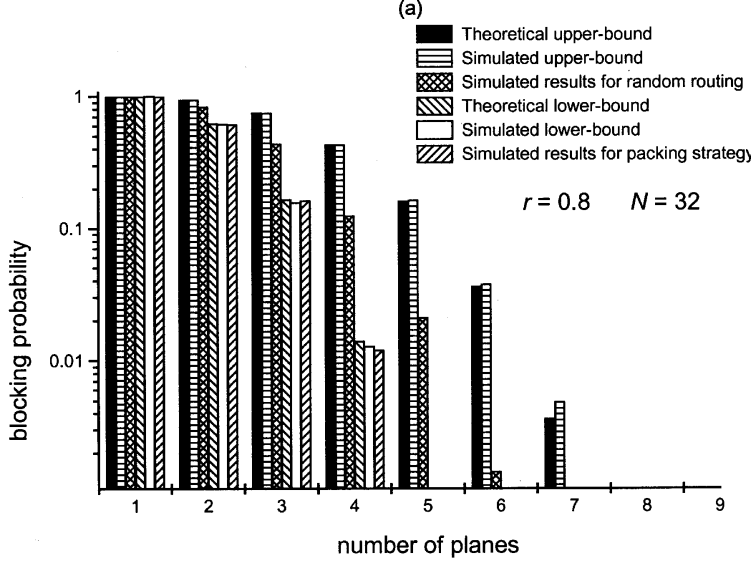

(b)

Fig. 5. Blocking probability of $32 \times 32$ VSOB network.

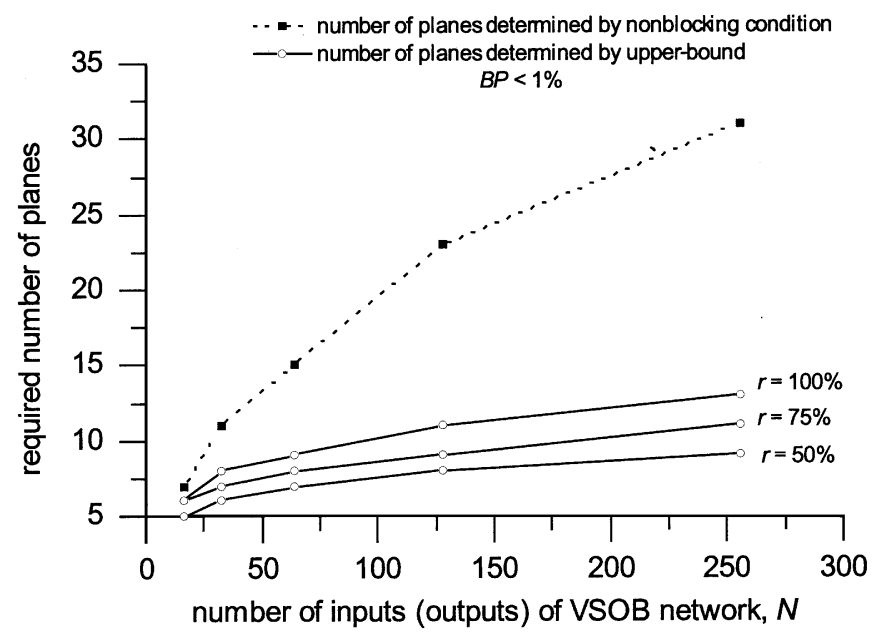

Fig. 6. Minimum number of planes for different size networks with different workloads.

For comparison, we also show in Fig. 6 the minimum numbers of planes given by the condition of strictly nonblocking [13]. For large networks, the results in Fig. 6 show that the hardware cost given by nonblocking condition is considerably higher than that given by the probabilistic analysis. For a switching network with $N=256$, the minimum number of planes given by the nonblocking condition is $m=2 \sqrt{N}-1=31$, whereas the minimum number of planes given by the upper bound in (6) is only 11 for $r=75 \%$ and $B P<1 \%$, so $(31-11) / 31 \cong 64.5 \%$ of the hardware can be reduced with only a very small sacrifice

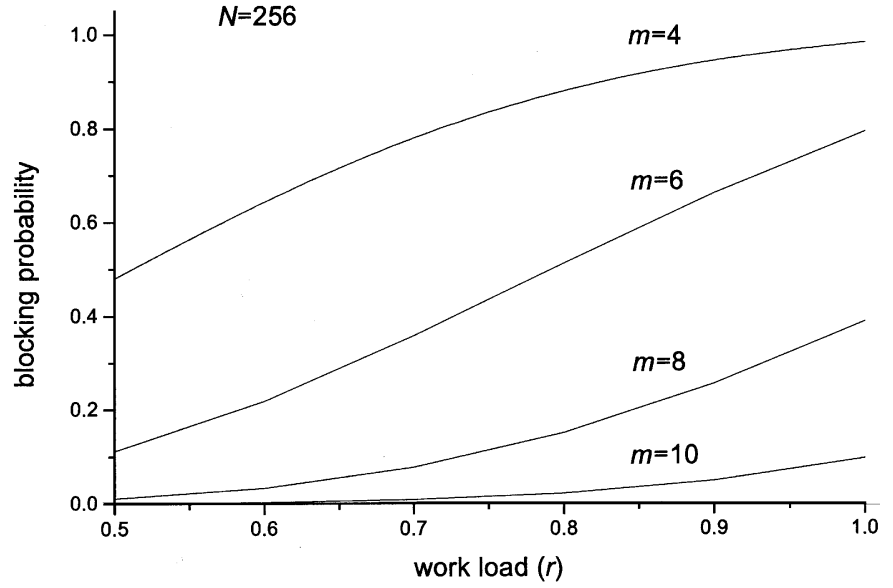

Fig. 7. Blocking probability versus the work loads for VSOB $(256, m)$ network.

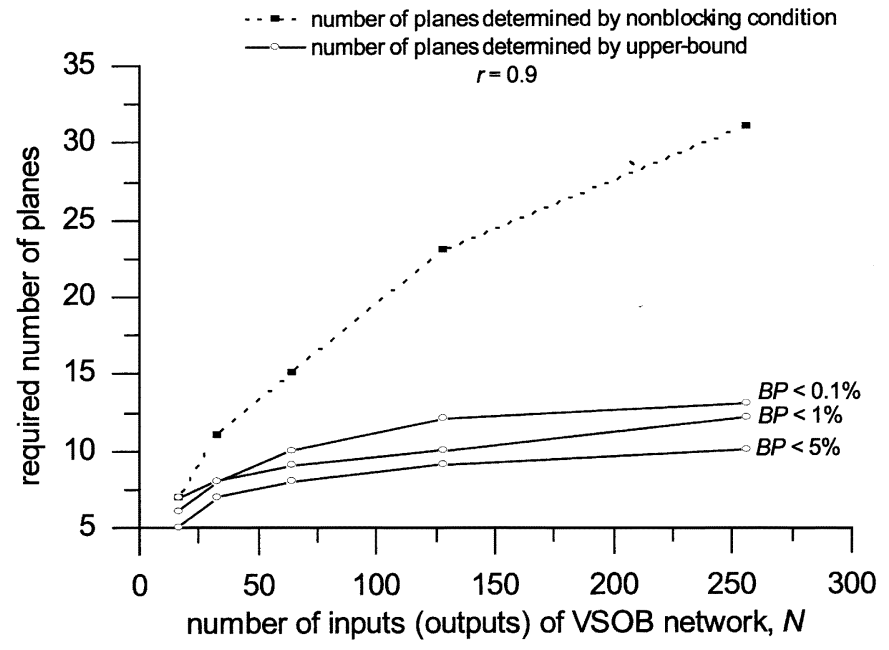

Fig. 8. Minimum number of planes for different size networks with different requirements of blocking probability.

in blocking probability of the network $(B P<1 \%)$. The results in Fig. 6 also indicate that for a given requirement of blocking probability, the hardware cost estimated by the upper bound is not very sensitive to the workloads. For a network with $N=128$ and $B P<1 \%$, the minimum number of planes estimated by the upper bound is 9 for $r=75 \%$ and 11 for $r=100 \%$, and all these numbers of planes are considerably less than the 23 planes as given by the nonblocking condition.

To find out further the effects of workload variations on blocking probability, we illustrate in Fig. 7 the blocking probability of VSOB $(256, m)$ against the workload $(r)$ for different number of planes.

It is interesting to note in Fig. 7 that although the blocking probabilities corresponding to different numbers of planes increase monotonically with the increase of workloads, but their sensitivities to workload changes are different. When workload varies from $60 \%$ to $90 \%$, the blocking probabilities of $\operatorname{VSOB}(256,4)$ varies from 0.64 to 0.94 and the blocking probabilities of $\operatorname{VSOB}(256,6)$ varies from 0.21 to 0.66 , respectively.

Fig. 8 illustrates more comparisons on the minimum number of planes estimated by our upper bound for different requirements of blocking probability. The results in Fig. 8 indicate 
that, for larger size networks, the hardware costs given by the nonblocking condition are still considerably higher than that given by the upper bound for a high requirement of blocking probability. For a switching network with $N=256$, the minimum number of planes given by the nonblocking condition is while the minimum number of planes given by the probabilistic analysis is only 13 for $r=90 \%$ and $B P<0.1 \%$, so the $(31-13) / 31 \cong 58 \%$ of the hardware can be reduced while a very low blocking probability is guaranteed $(B P<0.1 \%)$. We can observe from Fig. 8 that for a given workload, the hardware cost estimated by the bound is not sensitive to the requirements of blocking probability. For the network with $N=256$ and for $r=90 \%$, the minimum number of planes estimated by the upper bound is 12 for the requirement $B P<1 \%$ and 13 for the requirement $B P<0.1 \%$, and all these numbers of planes are considered less than the 31 planes required by the nonblocking condition.

\section{CONCLUSION}

Vertically stacked optical banyan (VSOB) networks are a class of attractive optical switching networks that usually require a high hardware cost to build. In this paper, we studied VSOB networks from probabilistic view and presented both upper and lower bounds on blocking probabilities of VSOB networks. These bounds can accurately describe the blocking behaviors of VSOB networks and they agree with the conditions of strictly nonblocking and rearrangeably nonblocking VSOB networks, respectively. Extensive simulation on a network simulator with both random routing and packing strategy has confirmed our bounds and shown that the blocking probability of packing strategy matches with the lower bound in a VSOB network. We can use the lower bound to estimate the blocking probability under packing strategy and also the minimum blocking probability of a VSOB network. On the other hand, our upper bound can guide the network designers to estimate the maximum blocking probability of a VSOB network in which different routing strategies may be applied. These bounds provide quantitative measurements for tradeoffs between network hardware cost and blocking probability, and show that network hardware cost can be dramatically reduced if a small and predictable blocking probability is allowed. This provides a significant tool for design of optical MINs at a desirable hardware cost and guaranteed performance with respect to blocking probability. The method of probabilistic analysis employed in this paper can be adopted for blocking probability analysis for other types of optical switching networks. Especially it can serve as a powerful tool for derivation of upper and lower bounds on blocking probability of optical networks. Note that the crosstalk-free constraint used in this paper may be too strict for some applications in which a certain degrees of crosstalk is allowed, and it is foreseeable that the hardware cost of networks can be reduced further for these applications. This will be our future research.

\section{ACKNOWLEDGMENT}

The authors would like to thank Editor N. McKeown and the anonymous reviewers for their valuable comments.

\section{REFERENCES}

[1] A. Jajszczyk and G. Jekel, "A new concept-Repackable networks," IEEE Trans. Commun., vol. 41, pp. 1232-1237, Aug. 1993.

[2] Y. Yang and J. Wang, "Wide-sense nonblocking Clos networks under packing strategy," IEEE Trans. Computer, vol. 48, pp. 265-284, Mar. 1999.

[3] V. R. Chinni et al., "Crosstalk in a lossy directional coupler switch," $J$. Lightwave Technol., vol. 13, pp. 1530-1535, July 1995.

[4] Y. Mun, Y. Tang, and V. Devarajan, "Analysis of call packing and rearrangement in multi stage switch," IEEE Trans. Commun., vol. 42, pp. 252-254, Feb./Mar./Apr. 1994.

[5] G. R. Goke and G. J. Lipovski, "Banyan networks for partitioning multiprocessor systems," in Proc. 1st Annu. Symp. Computer Architecture, 1973, pp. 21-28.

[6] H. S. Hinton, An Introduction to Photonic Switching Fabrics. New York: Plenum, 1993.

[7] X. Jiang, M. M. Khandker, and S. Horiguchi, "Nonblocking optical mins under crosstalk-free constraint," in Proc. IEEE Workshop High Performance Switching and Routing, May 2001, pp. 307-311.

[8] C. P. Kruskal and M. Snir, "The performance of multistage interconnection networks for multiprocessors," IEEE Trans. Commun., vol. 32, pp. 1091-1098, Dec. 1993.

[9] C.-T. Lea, "Multi- $\log _{2} N$ networks and their applications in high speed electronic and photonic switching systems," IEEE Trans. Commun., vol. 38, pp. 1740-1749, Oct. 1990.

[10] F. L. Thomson, Introduction to Parallel Algorithms and Architectures: Arrays, Trees, Hypercubes. San Mateo, CA: Morgan Kaufmann, 1992.

[11] G. Maier and A. Pattavina, "Design of photonic rearrangeable networks with zero first-order switching-element-crosstalk," IEEE Trans. Commun., vol. 49, pp. 1268-1279, July 2001.

[12] J. H. Patel, "Performance of processor-memory interconnections for multiprocessors," IEEE Trans. Comput., vol. C-30, pp. 771-780, Oct. 1981.

[13] M. M. Vaez and C.-T. Lea, "Strictly nonblocking directional-coupler-based switching networks under crosstalk constraint," IEEE Trans. Commun., vol. 48, pp. 316-323, Feb. 2000.

[14] —, "Wide-sense nonblocking banyan-type switching systems based on directional couplers," IEEE J. Select. Areas Commun., vol. 16, pp. 1327-1332, Sept. 1998.

[15] C. Y. Lee, "Analysis of switching networks," Bell Syst. Tech. J., vol. 34, no. 6, pp. 1287-1315, Nov. 1995.

[16] C. Jacobaeus, "A study on congestion in link systems," Ericsson Technics, vol. 51, no. 3, 1950

[17] C. Clos, "A study of nonblocking switching networks," Bell Syst. Tech. J., vol. 32, pp. 406-424, 1953.

[18] D. M. Dias and J. R. Jump, "Analysis and simulation of buffered delta networks," IEEE Trans. Comput., vol. C-30, pp. 273-282, Apr. 1981.

[19] A. Merchant, "Analytical models for the performance of banyan networks," Ph.D. dissertation, Comput. Sci. Dept., Stanford Univ., Stanford, CA, 1991.

[20] Y. Yang, "The performance of multicast banyan networks," J. Parallel Distributed Comput., vol. 60, pp. 909-923, 2000.

[21] Y. Yang and N. H. Kessler, "Modeling the blocking behavior of Clos networks," Int. J. Parallel Distrib. Syst. Netw., vol. 2, no. 1, pp. 1-9, 1999.

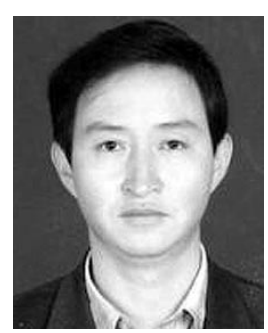

Xiaohong Jiang (M'03) received the B.S., M.S., and Ph.D. degrees from Xidian University, Xi'an, China, in 1989,1992 , and 1999 , respectively.

$\mathrm{He}$ is currently an Assistant Professor in the Graduate School of Information Science, Japan Advanced Institute of Science and Technology (JAIST). Dr. Jiang was a JSPS (Japan Society for the Promotion of Science) Post-Doctoral Research Fellow at JAIST from October 1999 to October 2001. He was a Research Associate in the Department of Electronics and Electrical Engineering, the University of Edinburgh, U.K., from March 1999 to October 1999. His research interests include interconnection networks, optical switch networks, WDM networks, IC yield modeling, timing analysis of digital circuits, clock distribution, and fault-tolerant technologies for VLSI/WSI. He has published over 30 technical papers in these areas. 


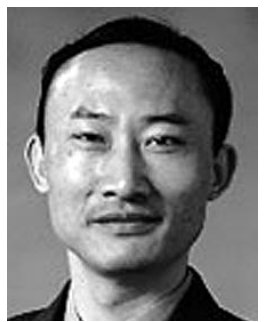

Hong Shen received the B.Eng. degree from Beijing University of Science and Technology, Beijing, China, the M.Eng. degree from the University of Science and Technology of China, and the Ph.Lic. and Ph.D. degrees from Abo Akademi University, Finland, all in computer science.

$\mathrm{He}$ is currently a full Professor in the Graduate School of Information Science, Japan Advanced Institute of Science and Technology, Ishikawa. Previously he was a Professor at Griffith University, Australia. He has published over 140 technical papers on algorithms, parallel and distributed computing, interconnection networks, parallel databases and data mining, multimedia systems, and networking. He has served as an Editor of Parallel and Distributed Computing Practice, Associate Editor of the International Journal of Parallel and Distributed Systems and Networks, a member of the editorial boards of Parallel Algorithms and Applications, International Journal of Computer Mathematics and the Journal of Supercomputing, and chaired various international conferences.

Dr. Shen is a recipient of the 1991 National Education Commission Science and Technology Progress Award and the 1992 Sinica Academia Natural Sciences Award.

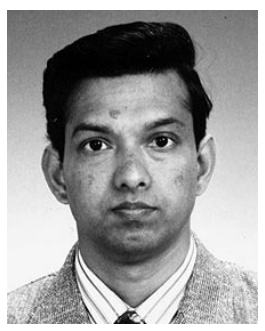

Md. Mamun-ur-Rashid Khandker (S'01) received the B.Sc. (Hons.) and M.Sc. degrees from Rajshahi University, Bangladesh. He is currently working toward the Ph.D. degree at the Japan Advanced Institute of Science and Technology (JAIST), Ishikawa.

After receiving his M.Sc. degree, he served as a faculty member in the Department of Applied Physics and Electronics, Rajshahi University. His research interests include interconnection networks, optical switch networks, and multiwavelength photonic packet switching. He is now working on multiwavelength address lookup for ultralarge optical networks. He holds one U.S. patent.

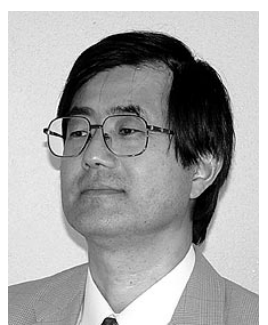

Susumu Horiguchi (S'79-M'81-SM'95) received the B.E., M.S., and Ph.D. degrees from Tohoku University, Tohoku, Japan, in 1976, 1978, and 1981, respectively.

He was a member of the faculty of the Department of Information Science, Tohoku University, from 1981 to 1992 . He was a Visiting Scientist at IBM Thomas J. Watson Research Center from 1986 to 1987. Since 1992, he has been a Professor in the Graduate School of Information Science at Japan Advanced Institute of Science and Technology (JAIST), Ishikawa, and has been conducting his research group as the chair of Multi-Media Integral System Laboratory at JAIST. He has been involved in organizing many international workshops, symposia, and conferences sponsored by IEEE, ACM, IASTED, IEICE, and IPS. His research interest has been mainly concerned with optical switch interconnection, interconnection networks, GRIDs computing, parallel computer architecture, and VLSI/WSI architecture. He is currently serving as an Editor for IEICE Transactions on Information and Systems and for the Journal of Interconnection Networks. 